\title{
Transfer as a function of shifts in task difficulty in verbal discrimination learning
}

\author{
YUNG CHE KIM, JAMES W. BROYLES, and MELVIN H. MARX \\ University of Missouri, Columbia, Missouri 65211
}

\begin{abstract}
This study investigated the relationship between transfer of training and shift in task difficulty for verbal discrimination learning. Also, it attempted to determine whether there are any differences in the tendency to make persistent errors in the two transfer conditions. Task difficulty was manipulated by varying the number of word alternatives, with either two words (easy task) or four words (hard task) in each word presentation set. Two lists, each with 26 sets of words, were presented for five trials. Each word set was shown for 1 sec, followed by $3 \mathrm{sec}$ for response and $1 \mathrm{sec}$ for feedback. A positive transfer effect in the hard-easy shift and a tendency toward negative transfer in the easy-hard shift were found.
\end{abstract}

Transfer of training from one level of task difficulty to another has been investigated in a number of studies. A common finding is that there is substantial positive transfer from the harder to the easier task and some negative transfer from the easier to the harder task. This result has been most often obtained for motor skills (Gibbs, 1954; Szafran \& Welford, 1950), but Green (1955) found no difference in a tracking task. Also, as Helson (1964) has pointed out, there is no basis for accepting the generality of the relationship, because opposite results have also been reported (e.g., by Bevan \& Saugstad, 1955, using a psychophysical weight discrimination task, and by Lawrence, 1952, working with visual discrimination in animals).

The primary purpose of this experiment was to investigate the relationship between transfer of training and the shift in task difficulty (e.g., hard to easy or easy to hard) for verbal discrimination learning (VDL). Positive transfer from VDL to paired associate learning has been reported (Battig \& Huang, 1971), and the effectiveness of prior learning-to-learn experience has been demonstrated (Mueller, Bamber, \& Lissa, 1973; Mueller, Hughes, \& Pickering, 1974). Positive transfer over several verbal lists, whether of the same or different type, has also been shown (Underwood, Shaughnessy, \& Zimmerman, 1972). However, the nature of the transfer effect resulting from shifts in difficulty in verbal materials remains to be determined. Thus, the particular experimental question with which this research

This work was supported in part by a Research Career Award from the National Institute of Mental Health and a grant from the Army Research Institute for Behavioral and Social Sciences, both to the third author. The opinions expressed herein are those of the authors and do not constitute endorsement by the U.S. Army. We wish to thank Shannon Young and Paulette LeClaire for assisting with this research and Charles J. Krauskopf for obtaining the aptitude measures. Yung Che Kim is Professor of Psychology, Keimyung University, Daegu, Republic of Korea. Requests for reprints should be addressed to Melvin H. Marx, Department of Psychology, University of Missouri, Columbia, Missouri 65211. is concerned is this: Does the same relationship as has been demonstrated for motor-skill learning (positive transfer for hard to easy shifts and negative transfer for easy to hard shifts) also hold for VDL?

A secondary objective of the research was to determine whether there are any differences in the tendency to make persistent, successive errors in the two transfer conditions. This tendency, labeled the stubborn-error effect (SEE), has been demonstrated in a number of previous verbal learning studies (e.g., Kim, Marx, \& Broyles, 1981; Marx, 1971; Marx \& Marx, 1980; Marx, Witter, \& Mueller, 1972).

\section{METHOD}

Subjects

The subjects were 17 male and 37 female students from the introductory psychology course at the University of MissouriColumbia. They were given credit for participating in the experiment as partial fulfillment of a course requirement. The subjects were divided into two basic treatment groups. Each group had 27 subjects, who completed two tasks, one of each difficulty condition. One other subject, whose data were incomplete, was discarded.

\section{Design and Materials}

Task difficulty was manipulated by varying the number of word alternatives in a set. The hard task utilized four words in each set, from which the randomly determined "correct" word had to be selected; the easy task had only two words in each set, one of which had been randomly determined to be "correct." Group H-E started with the hard $(\mathrm{H})$ task and then shifted to the easy (E) task, and the opposite shift in task difficulty occurred for Group E-H.

Both lists had three primacy and three recency buffer sets, none of which was scored. The remaining 20 sets of words provided data for scoring. All words were matched for approximately the same Thorndike-Lorge word frequency scores (1535 ), and the order in which these sets were presented was varied randomly across trials.

\section{Procedure}

Two lists, one of two-word and the other of four-word sets, were presented by slide projector (Kodak Ektagraphic Model AF-1) for five trials. Each word set was shown for $1 \mathrm{sec}$. Immediately after each word set, the subjects were allowed to 
respond for 3 sec. Responses were made by circling the number of the word selected on the answer sheet provided. The subjects were instructed to guess one "correct" word in each set on the first trial and to remember as many such correct responses as possible on the subsequent trials. A feedback period of $1 \mathrm{sec}$, during which the correct word of each word set was shown, was given after the response period and before the presentation of the next word set.

\section{RESULTS}

\section{Transfer Analysis}

Mean numbers of errors by groups and trials, along with transfer scores as a function of shifts in task difficulty, are presented in Table 1 . It is evident that a positive transfer effect did occur in Group H-E, and that there was a much smaller but consistent negative transfer effect in Group E-H; the latter effect was statistically reliable, however, only for the last trial. Positive transfer is indicated by positive differences, or fewer errors, and negative transfer by negative scores, or more errors.

These effects are described by the superior performance of Group H-E on the easy list, relative to Group E-H's level of performance on the same easy list when presented first, and by the slightly inferior performance of Group E-H on the hard list, again compared with the level of performance on the first presentation of that list, to Group H-E.

Although the subjects were randomly assigned to one of the two treatment groups, an additional check was made to ensure the equality of the groups on the basis of independently obtained aptitude and achievement measures. These measures were available for 36 of the 50 subjects, with an approximately even division between the two groups. Group E-H and Group H-E were compared on the following measures: high school rank; verbal, quantitative, and total scores on the School and College Aptitude Test; Missouri Mathematics Placement Test; and the Missouri College English Test. No consistent superiority was found for either group, and no difference between the group means approached statistical reliability.

\section{Stubborn-Error Effect Analysis}

The data were also analyzed to determine how the SEE was affected in the hard-easy and the easy-hard transfer conditions. We examined the number of subjects showing the SEE and the percent of error repetition as a function of the same error occurrence in the previous trial. The subjects were separated into two groups. All subjects who repeated the same error on at least two successive trials on either list were categorized as repeaters (R) and all others as nonrepeaters (NR). This categorization divided the subjects almost exactly in half.

A contingency analysis of the R vs. NR and E-H vs. $\mathrm{H}-\mathrm{E}$ variables showed that there were slightly more Rs than NRs in the two-word (easy) test when it preceded the four-word (hard) test (14 Rs compared with $13 \mathrm{NRs}$ ), but many fewer repeaters when it followed the four-word test (4 Rs compared with $23 \mathrm{NRs})\left(\chi^{2}=8.33, \mathrm{p}<.01\right)$. A similar contingency analysis revealed that there were more Rs than NRs in the four-word (hard) test when it followed the two-word (easy) test (22 Rs compared with $5 \mathrm{NRs)}$ and also when it preceded the two-word test (16 Rs compared with $11 \mathrm{NRs}$ ); the difference between these proportions was of marginal reliability $\left(\chi^{2}=3.20\right.$, $\mathrm{p}<.08)$.

The percentages of successive error repetitions over Trials 2-5 are shown, separately for the easy (twoword) and the hard (four-word) tasks, in Table 2. There was the usual regular increment in error repetition percentages over earlier trials as a function of prior

Table 1

Mean Number of Errors and Transfer Scores as a Function of Groups, Task Difficulty, and Trials

\begin{tabular}{|c|c|c|c|c|c|c|c|c|}
\hline & \multicolumn{8}{|c|}{ Task Difficulty } \\
\hline & \multicolumn{4}{|c|}{ Easy (Two Words) } & \multicolumn{4}{|c|}{ Hard (Four Words) } \\
\hline & Trial 2 & Trial 3 & Trial 4 & Trial 5 & Trial 2 & Trial 3 & Trial 4 & Trial 5 \\
\hline Group E-H & 5.70 & 2.22 & 1.41 & .63 & 8.33 & 4.93 & 4.19 & 2.63 \\
\hline Group H-E & 3.33 & .48 & .11 & .15 & 7.44 & 3.56 & 2.81 & 1.22 \\
\hline Transfer Score & $2.37 \dagger$ & $1.74 \dagger$ & $1.30^{*}$ & $48^{*}$ & -.89 & -1.37 & -1.38 & $-1.41 *$ \\
\hline
\end{tabular}

${ }^{*} p<.05 . \quad \dagger p<.01$.

Table 2

Percentage of Error Repetition (PE) as a Function of Prior Successive Occurrences of the Same Error

Task Difficulty

\begin{tabular}{|c|c|c|c|c|c|c|c|c|c|c|c|c|c|c|c|c|}
\hline & \multicolumn{8}{|c|}{ Easy (Two Words) } & \multicolumn{8}{|c|}{ Hard (Four Words) } \\
\hline & \multicolumn{2}{|c|}{ Trial 2} & \multicolumn{2}{|c|}{ Trial 3} & \multicolumn{2}{|c|}{ Trial 4} & \multicolumn{2}{|c|}{ Trial 5} & \multicolumn{2}{|c|}{ Trial 2} & \multicolumn{2}{|c|}{ Trial 3} & \multicolumn{2}{|c|}{ Trial 4} & \multicolumn{2}{|c|}{ Trial 5} \\
\hline & PE & $\mathbf{R}$ & $\mathrm{PE}$ & $\mathbf{R}$ & $\mathrm{PE}$ & $\mathbf{R}$ & $\mathrm{PE}$ & $\mathbf{R}$ & $\mathrm{PE}$ & $\mathbf{R}$ & $\mathrm{PE}$ & $\mathbf{R}$ & PE & $\mathbf{R}$ & $\mathrm{PE}$ & $\mathbf{R}$ \\
\hline Group E-H & 41 & 42 & 47 & 45 & 62 & 50 & 63 & 77 & 36 & 37 & 40 & 40 & 53 & 46 & 63 & 37 \\
\hline Group H-E & 32 & 39 & 48 & 50 & 53 & 0 & 57 & 0 & 39 & 30 & 44 & 34 & 50 & 45 & 54 & 29 \\
\hline
\end{tabular}

Note $-R=$ percentages for repeaters. 
successive repetitions of the same error, for both tasks. However, there was no difference between treatment groups.

Some indication of such a difference is provided, however, in another analysis. When an error occurred on the first-trial (guessing) response on the easy list of Group E-H, it was followed by $9 \%$ successive error repetitions (on more than two trials), compared with only $1 \%$ such repetitions on the easy list when that list followed the hard list (Group H-E); this difference was statistically reliable $(t=3.12, p<.01)$. The same measure after a correct response on Trial 1 (when errors did occur thereafter) showed 6\% repetitions of such repeated errors for Group E-H and, again, only $1 \%$ for Group H-E, but this difference was not statistically reliable. The same analysis performed on the hard list yielded more suggestive evidence of a kind of negative transfer (24\% error repetitions for Group E-H compared with $15 \%$ for Group H-E on the easy list, following a first-trial error, and $14 \%$ repetitions for Group E-H compared with $5 \%$ repetitions for Group H-E following a correct response on the first trial), but the differences were not statistically reliable.

\section{DISCUSSION}

The present results for VDL are clearly concordant with the motor-skill results cited earlier; positive transfer appears to occur when there is a shift from a relatively harder to an easier task, and some negative transfer is suggested on the relatively harder task when it follows the easier one, although there is only a little statistical support for the latter effect.

There is no basis in the present data for identifying the source of these differential results. Adequate interpretations can be advanced only after more analy tic research has indicated specific differences, as in cognitive strategies or memory mechanisms, between the conditions under comparison. Nevertheless, some suggestions pointing to such interpretations may be offered.

The difference obtained in the SEE measure following an incorrect guess on Trial 1 is suggestive. Why were Group E-H subjects more committed to such erroneous guesses than were Group H-E subjects? One possibility is that they were simply less motivated, having been started on the less difficult task. Put another way, they may have been less "upwardly adapted," as Heim (1957) suggested in her evaluation of performance effects related to shifting levels of task difficulty.

It might also be useful to develop Macrae and Holding's (1965) notion of differential commitment to erroneous responses. Independent manipulation of the determinants of such commitment could then be arranged in conjunction with varying levels of task difficulty, and the transfer effects could be measured.

While definitive interpretations are obviously premature at this time, the present data not only offer additional empirical support for the earlier difficulty-transfer relationship found in motor-skill research but also offer some suggestions for future research on this important but little researched problem.

\section{REFERENCES}

Battig, W. F., \& Huang, S. Experiment giant: Effects of five sources of variation in intratask interference on paired-associate learning and transfer. Psychonomic Monograph Supplements, 1971, 4(Whole No. 57), 165-187.

Bevan, W., \& Saugstad, P. Breadth of experience, ease of discrimination, and efficiency of generalization. British Journal of Psychology, 1955, 46, 13-19.

GibBs, C. B. Transfer of training and skill assumptions in tracking tasks. Quarterly Journal of Experimental Psychology, 1954, 3, 99-100.

Green, R. F. Transfer of skill on a following tracking task as a function of task difficulty (target size). Journal of Psychology, 1955, 39, 355-370.

Heim, A. W. Psychological adaptation as a response to variations in difficulty and intensity. Journal of General Psychology, 1957, 56, 193-211.

Helson, H. Adaptation-level theory: An experimental and systematic approach to behavior. New York: Harper \& Row, 1964.

Kim, Y. C., Marx, M. H., \& Broyles, J. W. The stubbornerror effect in verbal discrimination learning. Bulletin of the Psychonomic Society, 1981, 18, 5-8.

LAwrence, D. H. The transfer of a discrimination along a continuum. Journal of Comparative and Physiological Psychology, 1952, 45, 511-516.

Macrae, A. W., \& Holding, D. H. Guided practice in direct and reversed serial tracking. Ergonomics, 1965, 8, 487-492.

MARX, M. H. Increased probability of error repetition as a function of number of successive prior repetitions. Perceptual and Motor Skills, 1971, 32, 544-546.

Marx, M. H., \& MARX, K. Confirmation of the stubborn-error effect in human multiple-choice verbal learning. Bulletin of the Psychonomic Society, 1980, 16, 477-479.

Marx, M. H., WitTer, D. W., \& Mueller, J. H. Additional data on probability of error repetition following varying numbers of successive prior repetitions. Perceptual and Motor Skills, 1972,34, 525-526.

Muelle R, J. H., Bamber, R. T., \& Lissa, D. J. Learning to learn in verbal discrimination learning with single- and doublefunction lists. Journal of Experimental Psychology, 1973, 97, 182-188.

Mueller, J. H., Hughes, C. W., \& Pickering, J. P. Total time and learning to learn in paired-associate and verbal-discrimination tasks. American Journal of Psychology, 1974, 87, 107-116.

Szafran, J., \& Welford, A. T. On the relation between transfer and difficulty of initial task. Quarterly Journal of Experimental Psychology, 1950, 2, 88-94.

Underwood, B. J., Shaughnessy, J. J., \& Zimmerman, J. Learning-to-learn verbal discrimination list. Journal of Verbal Learning and Verbal Behavior, 1972, 11, 96-104.

(Received for publication June 27, 1981.) 